\title{
Improving Safety for Driverless City Vehicles: Real-Time Communication and Decision Making
}

\author{
Andrei Furda ${ }^{1}$, Laurent Bouraoui ${ }^{2}$, Michel Parent ${ }^{2}$, and Ljubo Vlacic ${ }^{1}$ \\ ${ }^{1}$ Intelligent Control Systems Laboratory (ICSL) \\ Institute of Integrated and Intelligent Systems \\ Griffith University \\ Brisbane, Australia \\ ${ }^{2}$ Institut National de Recherche en Informatique et et Automatique (INRIA) \\ Team IMARA \\ 78153 Le Chesnay, France \\ a.furda@griffith.edu.au; laurent.bouraoui@inria.fr; michel.parent@inria.fr; 1 .vlacic@ griffith.edu.au
}

\begin{abstract}
This paper elaborates on the Cybercars-2 Wireless Communication Framework for driverless city vehicles, which is used for Vehicle-to-Vehicle and Vehicle-to-Infrastructure communication. The developed framework improves the safety and efficiency of driverless city vehicles. Furthermore, this paper also elaborates on the vehicle control software architecture. Onroad tests of both the communication framework and its application for real-time decision making show that the communication framework is reliable and useful for improving the safe operation of driverless city vehicles.
\end{abstract}

Index Terms-close proximity vehicle-to-vehicle (V2V) communication, vehicle-to-infrastructure (V2I) communication, driverless city vehicles, real-time decision making

\section{INTRODUCTION}

Driverless city vehicles' ability to communicate is crucial for enabling their cooperative driving maneuvers [1]-[3], and for improving their safety and efficiency. Vehicle to-Vehicle (V2V) communication leads to improved safety by allowing nearby vehicles to exchange relevant information about their traffic environment (e.g. potential hazards, accidents, etc.), and/or about their driving intentions. In addition, Vehicle-toInfrastructure (V2I) communication can improve the road network's efficiency and reduce pollution by informing driverless vehicles about traffic conditions (e.g. traffic congestions, alternative routes, etc.).

Worldwide standardization efforts, addressing the requirements for $\mathrm{V} 2 \mathrm{I}$ and $\mathrm{V} 2 \mathrm{~V}$ communication, are in progress. One of the most significant efforts is ISO TC204 WG16 and its CALM (Continuous Air Interface for Long and Medium Range) concept. CALM is a set of standards which provides protocols and parameters, specifically addressing the requirements for ITS communication [4].

Real-time decision making for driverless city vehicles has been recognized as a major research topic which needs to be further addressed. Various real-time decision making approaches have been applied in the DARPA Urban Challenge 2007 [5], such as finite automata (e.g. vehicles "Junior" [6], "Odin" [7], "AnnieWAY" [8]), reasoning (e.g. vehicle "Knight Rider" [9]), heuristic decision rules (e.g. vehicle "Skynet"
[10]), or weighted votes (e.g. vehicle "Caroline" [11]). Nevertheless, the winners of the DARPA Urban Challenge 2007 (vehicle "Boss" [12]) noticed that their traffic representation was not sufficient to make intelligent driving decisions comparable to human drivers.

The application of $\mathrm{V} 2 \mathrm{~V}$ communication in order to improve the driverless city vehicle's real-time decision making abilities is a relevant research topic in this field. This paper elaborates on the Cybercars-2 Communication Framework (Section II), gives an overview about the ICSL Vehicle Control Software (Section III), and presents experimental results of both the communication framework's performance and its application for real-time decision making (Section IV). Section V concludes this paper.

\section{CYBERCARS-2 COMMUNICATION FRAMEWORK}

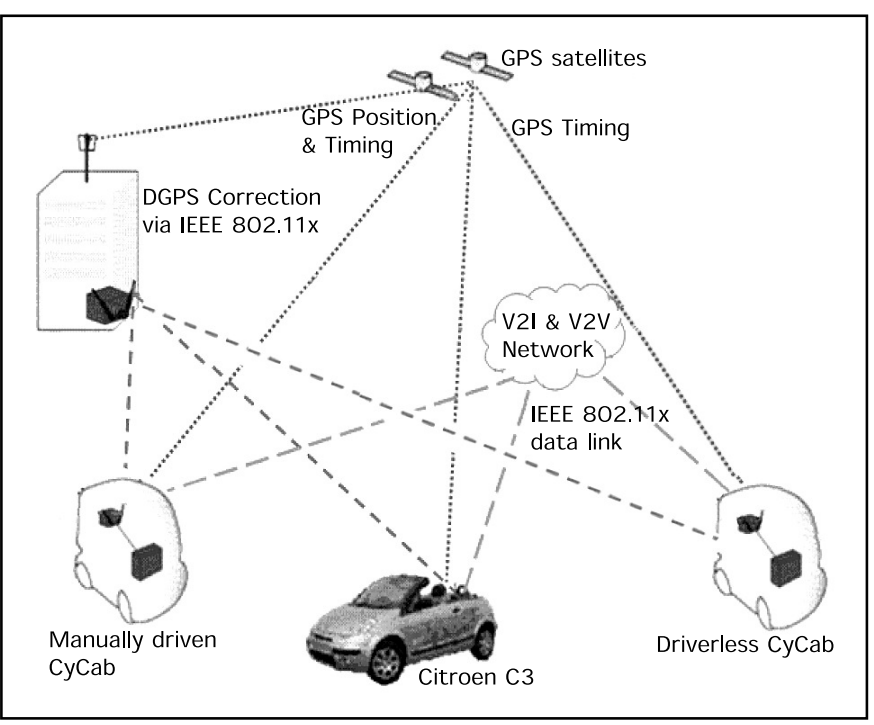

Figure 1. Overview of the example communication setup (adapted from [4]). The driverless CyCab communicated with a manually driven CyCab and a conventional car. 
The main objective of the Cybercars-2 Communication Framework [4] is to enable autonomous vehicles to safely perform cooperative driving maneuvers. Cybercars are Intelligent Transportation Systems based on road vehicles with fully automated driving capabilities [13], [14]. In the current stage, Cybercars are not intended to operate in public traffic, but in restricted environments, such as airports or theme parks.

Fig. 1 shows an overview of the Cybercars-2 communication setup. The example setup consists of three communicating vehicles: a driverless $\mathrm{CyCab}$ [15], [16], a human driven $\mathrm{CyCab}$ and a conventional car (Citroen $\mathrm{C} 3$ ) equipped with an Advanced Driver Assistance System.

The communication framework consists of five layers: Physical Layer, MAC Layer, Network Layer, System Service Layer, and Application Layer (Fig. 2).

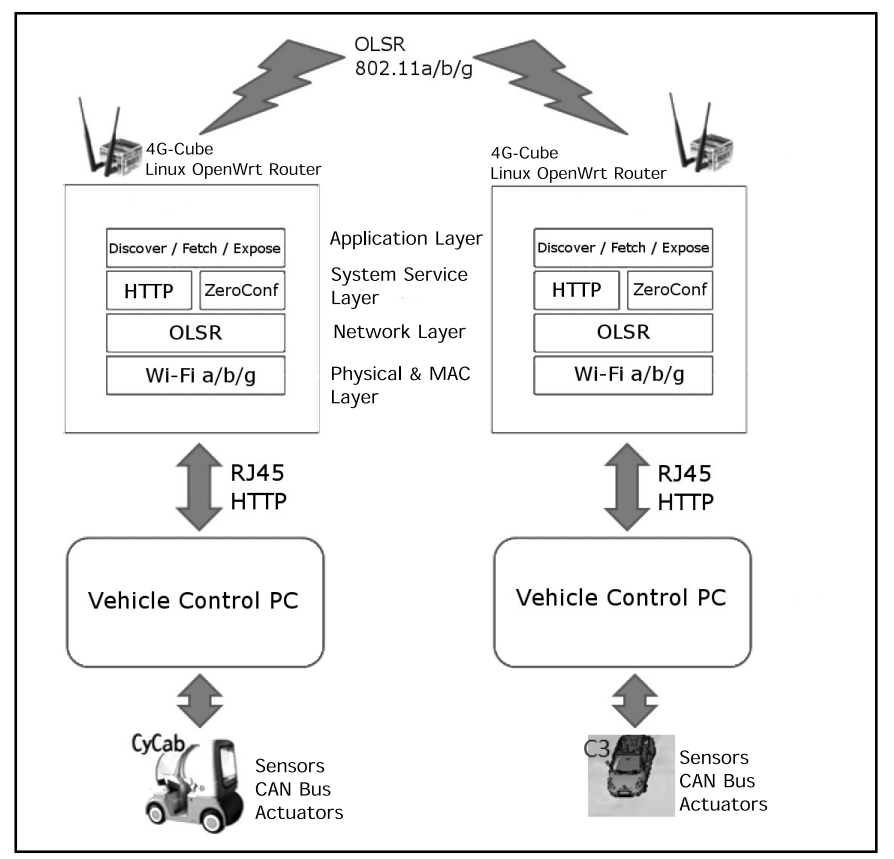

Figure 2. The Cybercars-2 Communication Framework architecture.

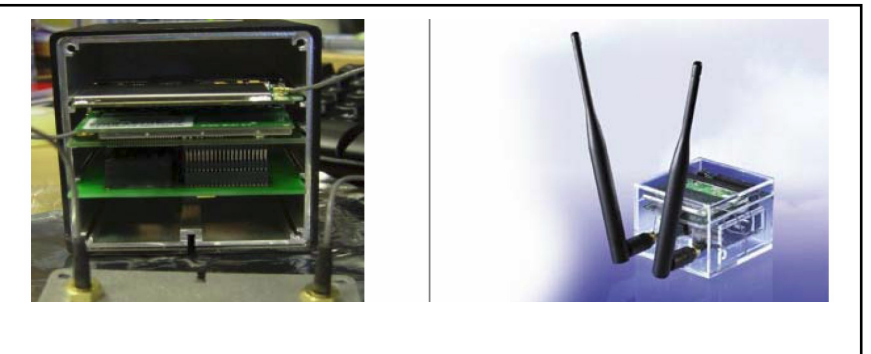

Figure 3. The 4G-Cube is a small MIPS-based computer with integrated Wi$\mathrm{Fi}(\mathrm{b} / \mathrm{g})$ and Ethernet interfaces [4].

\section{A. Physical and MAC Layers}

The Cybercars-2 Communication Framework accommodates the use of the following three communication standards/recommendations: IEEE $802.11 \mathrm{p}$, IEEE $802.11 \mathrm{a} / \mathrm{b} / \mathrm{g}$ and WWAN technologies, such as GPRS, UMTS or WiMAX.
The IEEE 802.11p recommendation is used for V2V/V2I communication, IEEE $802.11 \mathrm{a} / \mathrm{b} / \mathrm{g}$ for support information, and WWAN to monitor the traffic flow and to improve its efficiency.

At the current stage, communication equipment compliant to IEEE $802.11 \mathrm{~b} / \mathrm{g}$ is used for $\mathrm{V} 2 \mathrm{~V}$ and V2I communication. Therefore, the MAC Layer includes the functionalities which are available for the commercial IEEE 802.11 compliant equipment. The hardware and software enabling communication is integrated into the 4G-Cube (Fig. 3), a MIPS-based computer running Linux.

\section{B. Network Layer}

In order to fulfill the main objective, which is to enable cooperative maneuvers, the focus is mainly on close proximity communication between nearby vehicles. In this highly dynamic application environment, dynamic routing is a major requirement. For this purpose, the Optimized Link State Routing protocol (OLSR) is used. The OLSR protocol provides the functionality required for vehicle communications. It was designed specifically for multihop networks with a strong and efficient mechanism for data flow delivery, and it enables the quick creation and reconfiguration of vehicle mesh networks [17]. OLSR operates as a table driven, proactive protocol. It regularly exchanges topology information with other nodes of the network. Each node selects a set of its neighbour nodes as "multipoint relays" (MPR). Only MPR nodes are responsible for forwarding control traffic, which is intended to be delivered over the entire network. MPRs provide an efficient mechanism to control traffic flooding by optimizing the number of transmissions required, and therefore it helps to avoid network overload.

\section{System Service Layer}

As part of the system service layer, the service discovery mechanism "Zeroconfiguration" from Apple has been adopted, as it helps to improve the network establishment procedure. Multicast DNS (mDNS) is used to provide a local namespace in order to abstract the vehicle's network addresses. On top of DNS (or mDNS), Service Discovery (DNS-SD) can be used by the 4G-Cube to publish or query information about the applications or services running in the neighbourhood [4].

\section{Application Layer}

The communication protocol is based on the HTTP 1.1 protocol and uses the HTTP GET and POST requests. Three functions are provided: discover (to list available services), expose (to send data to all network nodes), fetch (to receive data).

\section{VEHICLE CONTROL SOFTWARE}

The driverless vehicle control software consists of the following main modules: the World Model, the Real-Time Decision Making \& Driving Maneuver Control, and the Vehicle Interface (Fig. 4).

The World Model represents the driverless vehicle's view of its road environment. This software module merges a priori 
given information (e.g. known intersections, maps) with traffic features which are perceived during the vehicle's movement using on-board sensors and/or data received through communication with other vehicles.

As part of the World Model, the Perception Module includes the functionalities for accessing data from on-board sensors (e.g. GPS, Lidar), but also for retrieving data through communication (e.g. 4G-Cube).

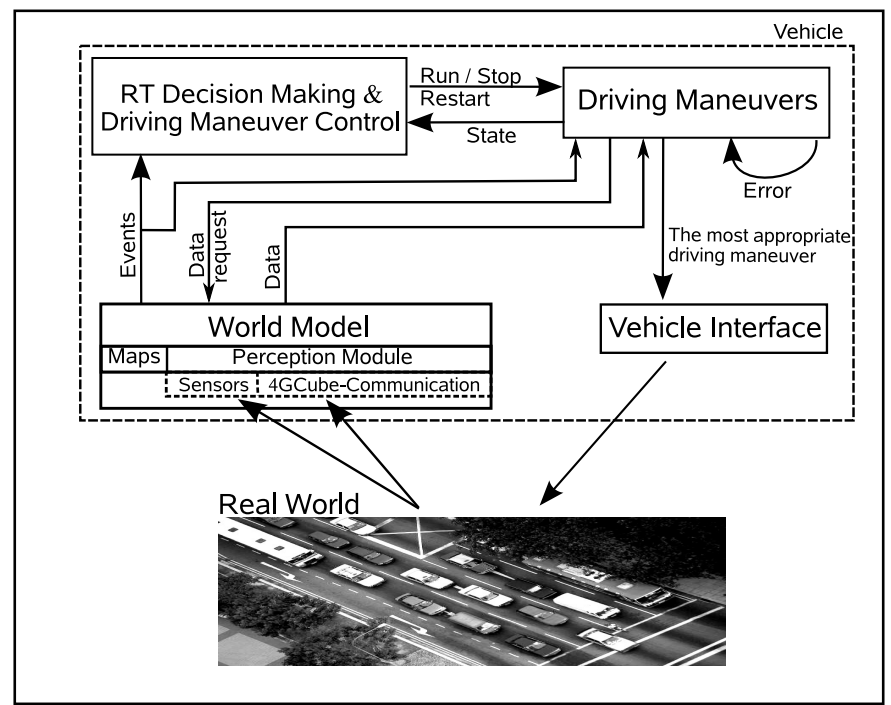

Figure 4. The ICSL driverless vehicle control software architecture.

The data contained in the World Model is constantly updated in real-time. The main purpose of the World Model within the vehicle control software is to provide the Real-Time Decision Making \& Driving Maneuver Control module, with accurate information about the vehicle's environment. This is accomplished in two ways:

- by actively notifying registered modules when the status of World Model Events changed (implemented using the Observer design pattern [18]), and

- by allowing other modules to access all information stored in the World Model structure.

Based on the information received from the World Model, the Real-Time Decision Making \& Driving Maneuver Control module makes real-time driving decisions. It decides and activates the most appropriate driving maneuver. Each driving maneuver is a closed-loop control algorithm, able to maneuver the vehicle in a specific traffic situation. Details about the decision making module have been published in [19]. The decision making module directs its output to the Vehicle Interface module.

Communication (V2V, but also V2I) is important for realtime decision making. In our experiments, we used $\mathrm{V} 2 \mathrm{~V}$ communication as the only source of information in order to test the decision making approach [19].

\section{EXPERIMENTAL RESULTS}

This section is structured as follows. The first subsection presents the results of experimental tests conducted by INRIA, and addresses the performance of the Cybercars-2 Communication Framework. The second subsection presents results of real-time decision making experiments. These experiments were conducted using the ICSL driverless vehicle control software, however, integrating V2V communication using the Cybercars-2 Communication Framework.

\section{A. Basic Communication Tests}

In order to prove the usability of the developed communication framework in real-world applications, INRIA conducted several experiments using two communicating $\mathrm{CyCab}$ vehicles (Fig. 5). Both vehicles were equipped with a 4G-Cube and used the software RTMaps to record communication data along with data from the vehicle's onboard DGPS receivers. The recorded data could be later replayed and analyzed.

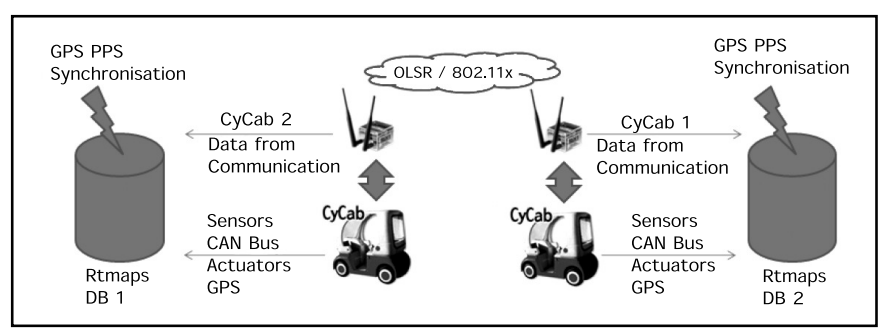

Figure 5. The communication experiment setup (adapted from [4]).

The main objective was to measure the delay of communication at various distances between the moving vehicles, and the maximum communication range. For this purpose, the distance error ERR_DIST is defined as:

$$
\begin{aligned}
& E R R \_D I S T=\operatorname{distance}(P o s 2-P O S 1)- \\
& \text { distance(PosCOM2 - PosCOM1), }
\end{aligned}
$$

where $\operatorname{Pos}\{n\}$ denotes the measured GPS position of vehicle $n$ (on-board sensor), and $\operatorname{PosCOM}\{\mathrm{n}\}$ denotes the received position of vehicle $\mathrm{n}$ (communication).

Any deviation from zero of ERR_DIST is caused by communication delays. Therefore, this variable is ideal to measure the complete delay in the communication system, including the delays caused by the communication server and the parsing of data structures.

Fig. 6 shows the distance error ERR_DIST in relation to the vehicle's distance in a first experiment. The scenario of this first experiment was the crossing of an intersection. In the second experiment (Fig. 7), the vehicles started front to front in a distance out of communication range and crossed each other. The graph shows that the communication was established and maintained when the distance between the vehicles was less than around 140 meters. 


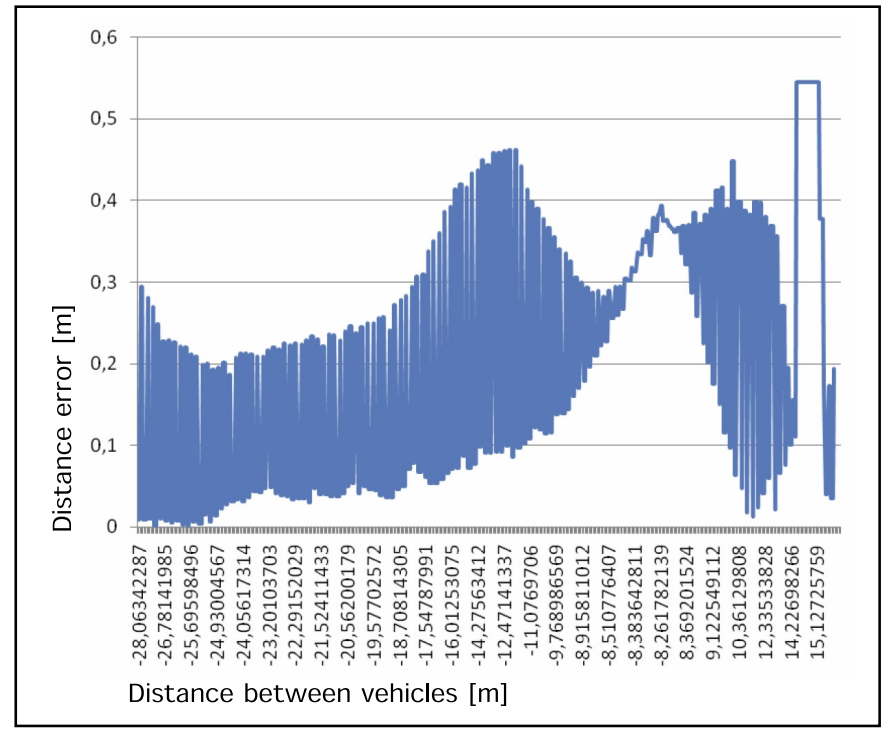

Figure 6. Distance error resulting from communication delays (source: [4]). In this experiment, the vehicles were crossing an intersection.

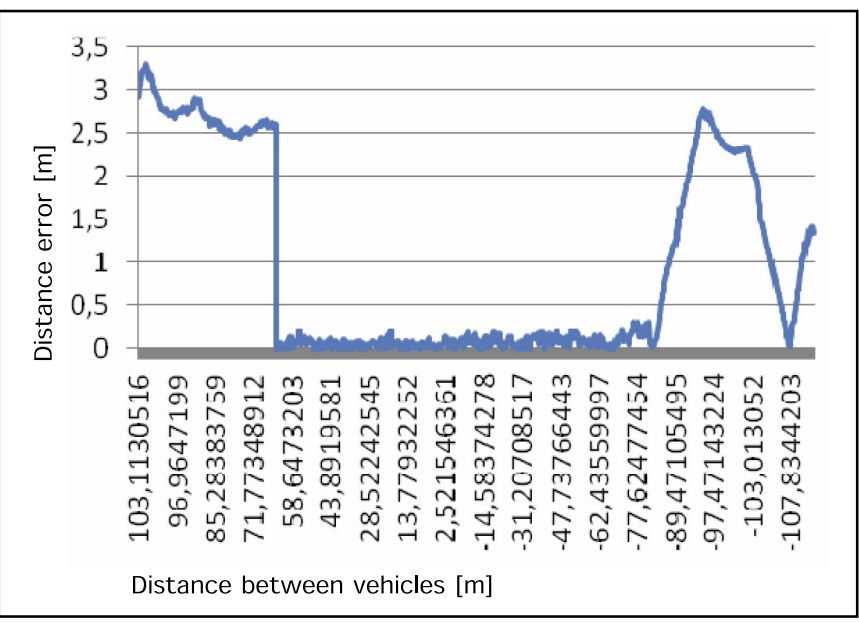

Figure 7. Distance error for two approaching vehicles (source: [4]).

\section{B. Communication-Based RT Decision Making Experiments}

Experimental tests have been carried out, which show not only the communication performance, but also the application of communication for real-time decision making. For these experiments we used a driverless vehicle (Cycab, manufactured by Robosoft, France), a second, manually driven Cycab and a conventional car (Citroen C3). All vehicles, sensors, and test facilities haven been provided by the French research institute INRIA (team IMARA). The decision making approach [19] has been developed at ICSL, Griffith University, Australia.

All vehicles, including the conventional car, were equipped with differential GPS (DGPS) and were able to communicate over the communication infrastructure. In addition to its own GPS position, the driverless vehicle was able to receive the GPS positions of the other two vehicles.

Furthermore, the driverless vehicle's world model included a priori information, such as the position of intersections and positions of imaginary stop signs. In order to test the decision making approach, three different traffic scenarios have been set up, all showing a common decision situation: passing a stopped vehicle under different traffic conditions.

1) Experiment 1: In the first traffic scenario, the driverless vehicle approached a stopped vehicle. Safe passing was possible, and the oncoming traffic lane was free of any obstacles (Fig. 8). In this first scenario, the driverless vehicle immediately started the passing maneuver when it approached the stopped vehicle.

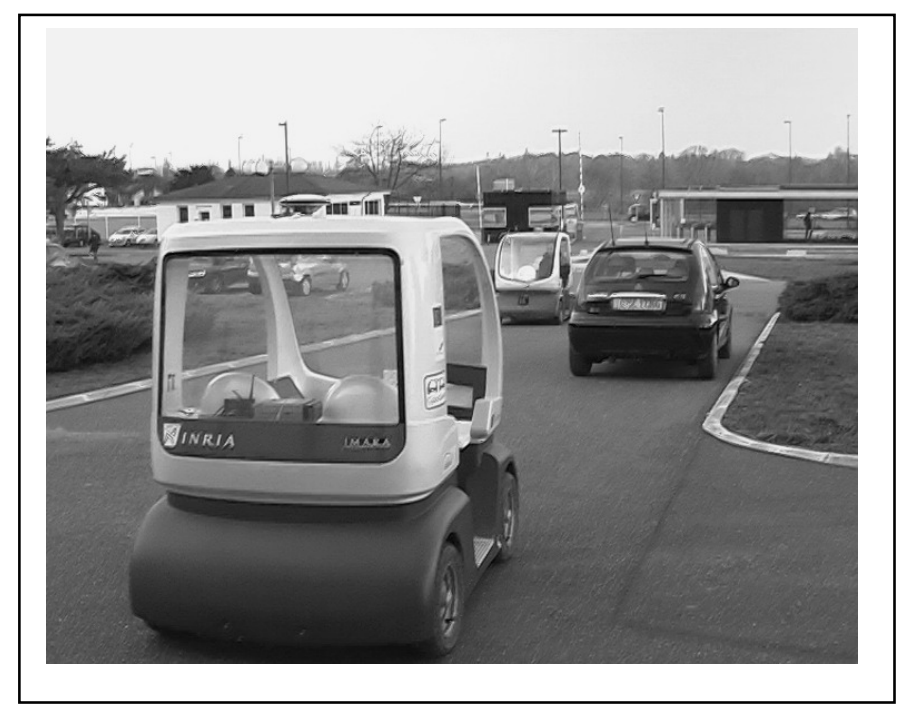

Figure 8. Experiment 1: the driverless vehicle passes a stopped vehicle.

2) Experiment 2: The second traffic scenario was similar to the first, however another manually driven vehicle was oncoming, making safe passing impossible. In this second scenario, the driverless vehicle waited behind the stopped vehicle, and started passing the stopped vehicle when the oncoming traffic lane was free.

3) Experiment 3: In the third traffic scenario, a manually driven vehicle was stopped at an intersection. The driverless vehicle waited behind the stopped vehicle until it crossed the intersection. Then the driverless vehicle continued driving, stopped at the imaginary stop sign before continuing across the intersection.

The main goal of the experiments related to real-time decision making was to demonstrate that the ICSL driverless vehicle control software, and most of all the ICSL real-time decision making approach [19] works with real vehicles and real sensors and meets the real-time decision making requirements. The integration of the Cybercars-2 Communication Framework developed by INRIA into the ICSL control software gave us the first opportunity to test the decision making approach under real-world conditions, while at the same time proving the usability of the Cybercars-2 Communication Framework.

During the experimentation phase at INRIA, all experiments were repeated numerous times. Often, new software or hardware related problems were detected and 
solved. Consequently, these experimental results cannot be regarded as a rigorous test benchmark for the quality of the entire system.

Table I lists the number of successful and failed repetitions during the conducted experiments. These results were recorded after the software and hardware systems reached a sufficient reliability for our test purposes. Although there were a number of remaining problems, such as the unreliable execution of driving maneuvers, which could not be solved in the available time frame, the results show that in all repeated experiments, the decision making module was always able to avoid collisions with other vehicles and make appropriate driving decisions in real-time. Consequently, as real-time decision making was purely based on communication, the results also show that the used communication framework proved to be sufficiently reliable, and is therefore useful for improving the safe operation of driverless vehicles.

TABLE I. RESULTS OF REAL-TIME DECISION MAKING EXPERIMENTS.

\begin{tabular}{|c||c|c|c||c|c|}
\hline Experiment & \#trials & \multicolumn{2}{c||}{ Decision Making } & \multicolumn{2}{c|}{ Driving Maneuvers } \\
\hline & & Successful & Failed & Successful & Failed \\
\hline \hline Experiment 1 & 17 & $17^{\mathrm{a}}$ & 0 & 9 & $8^{\mathrm{b}}$ \\
\hline Experiment 2 & 13 & $13^{\mathrm{c}}$ & 0 & 7 & $5^{\mathrm{d}, \mathrm{e}}$ \\
\hline Experiment 3 & 12 & $12^{\mathrm{f}}$ & 0 & 7 & $5^{\mathrm{g}}$ \\
\hline
\end{tabular}

aThe decision to pass was made correctly and the passing maneuver was activated.

bThe driving maneuver for passing was not correctly implemented.

cThe decision to pass when possible was correctly made.

$\mathrm{d}$ The execution of the passing driving maneuver failed.

eOne recorded experiment included only the decision making and no subsequent driving maneuvers.

fThe decision to stop and wait behind the stopped vehicle was correctly made

gThe driving maneuver for continuing when the intersection was free failed.

\section{CONCLUSION}

The first part of this paper has presented the Cybercars-2 Communication Framework. The communication framework enables Vehicle-to-Vehicle (V2V) and Vehicle-toInfrastructure (V2I) communications for driverless city vehicles, which improve the safety and efficiency. The second part of this paper has presented an overview about the ICSL driverless vehicle control software, its architecture and integration of communication modules. The modular objectoriented software design, a modular World Model and the flexible real-time decision making approach enables the integration and use of traffic environment information from various sources, such as communication with other vehicles.

The third part of this paper has presented experimental results for both the Cybercars-2 Communication Framework and its application for real-time decision making. The real-time decision making approach based only on $\mathrm{V} 2 \mathrm{~V}$ communication has been tested in three different traffic scenarios, where the decision regarding passing a stopped vehicle was safety crucial.
The experimental results show that the ICSL vehicle control software, including the developed real-time decision making approach, in combination with the CybercarsCommunication Framework, provide a reliable solution for improving the safe operation of driverless city vehicles.

\section{REFERENCES}

[1] J. Baber, J. Kolodko, T. Noel, M. Parent, and L. Vlacic, "Cooperative autonomous driving - intelligent vehicles sharing city roads," IEEE Robotics \& Automation Magazine, March 2005

[2] J. Kolodko and L. Vlacic, "Cooperative autonomous driving at the Intelligent Control Systems Laboratory," IEEE Intelligent Systems, vol. 18 , no. 4, pp. 8-11, 2003

[3] R. Naumann and R. Rasche, "Intersection collision avoidance by means of decentralized security and communication management of autonomous vehicles," in 30th ISATA Conference on ATT/ITS, Florence, Italy, 1997.

[4] B. Molinete, L. Bouraui, E. Naranjo, H. Kostense, F. Hendriks, J. Alonso, R. Lobrino, and L. Isasi, CyberCars-2: Close Communications for Cooperation between CyberCars. Technical Report Project No IST-2004-0228062, 2009.

[5] DARPA, "Urban challenge rules," 2006. [Online]. Available: http://www.darpa.mil/grandchallenge/docs/ Urban Challenge Rules 121106.pdf

[6] M. Montemerlo, J. Becker, S. Bhat, H. Dahlkamp, D. Dolgov, S. Ettinger, and D. Haehnel, "Junior: The stanford entry in the urban challenge," Journal of Field Robotics, vol. 25, no. 9, 2008.

[7] A. Bacha, C. Bauman, R. Faruque, M. Fleming, and C. Terwelp, "Odin: Team victortangos entry in the darpa urban challenge," Journal of Field Robotics, vol. 25, no. 8, 2008.

[8] S. Kammel, J. Ziegler, B. Pitzer et al, "Team annieways autonomous system for the 2007 darpa urban challenge," Journal of Field Robotics, vol. 25, no. 9, 2008.

[9] B. J. Patz, Y. Papelis, R. Pillat, G. Stein, and D. Harper, "A practical approach to robotic design for the darpa urban challenge," Journal of Field Robotics, vol. 25, no. 8, 2008.

[10] I. Miller, M. Campbell, D. Huttenlocher, and F.-R. Kline, "Team cornells skynet: Robust perception and planning in an urban environment," Journal of Field Robotics, vol. 25, no. 8, 2008.

[11] F. W. Rauskolb, K. Berger, C. Lipski et al, "Caroline: An autonomously driving vehicle for urban environments," Journal of Field Robotics, vol. 25, no. 9, 2008.

[12] C. Urmson et al., "Autonomous driving in urban environments: Boss and the urban challenge," Journal of Field Robotics, vol. 25, no. 8, 2008.

[13] M. Parent and G. Gallais, "Intelligent transportation in cities with cts" in ITS World Congress, Chicago, USA, 2002.

[14] M. Parent and G. Gallais, "Cybercars: Review of first projects," in Ninth International Conference on Automated People Movers, Singapore, 2003.

[15] G. Baille, P. Garnier, H. Mathieu, and P.-G. R., "Le cycab de l'inria rhone-alpes," Intitut National de Recherce en Informatique et en Automatique (INRIA), France, Tech. Rep. 0229, 1999.

[16] C. Pradalier, J. Hermosillo, C. Koike, C. Braillon, P. Bessire, and C. Laugier, "The cycab: a car-like robot navigating autonomously and safely among pedestrians," Robotics and Autonomous Systems, vol. 50, no. 1, pp. 51-68, 2005.

[17] T. Clausen, P. Jacquet, A. Laouiti, P. Muhlethaler, A. Qayyum, and L. Viennot, "Optimized link state routing protocol," in IEEE INMIC, 2001.

[18] E. Gamma, R. Helm, R. Johnson, and J. Vlissides, Design Patterns: elements of reusable object-oriented software. Addison-Wesley, 1994.

[19] A. Furda and L. Vlacic, "Towards increased road safety: Real-time decision making for driverless city vehicles," in 2009 IEEE International Conference on Systems, Man, and Cybernetics, San Antonio, TX, USA, 2009. 